A DFT Study of the Properties of $\mathbf{N}-\mathbf{H} \cdots \mathbf{N}$, Non-cooperativities, and Intermolecular Interactions in Linear trans-Diazene Clusters up to Ten Molecules

Hua-Jie Song ${ }^{*}, a, b \quad$ He-Ming Xiao ${ }^{a}$ and Hai-Shan Dong ${ }^{b}$

${ }^{a}$ Department of Chemistry, Nanjing University of Science and Technology., Nanjing 210094, China. Institute of Chemical Materials, China Academy of Engineering Physics, Mianyang 621900, China

E-mail: hjsongmoru@tom.com

TABLE 1. Lengths of $\mathrm{N}-\mathrm{H}$ bonds $(\AA)$ and charge transfers of the trans-diazene clusters $(n=2-10)$ at the B3LYP/6-31++G(d,p) level

\begin{tabular}{|c|c|c|c|c|c|c|c|c|c|c|c|}
\hline$n$ & \multicolumn{2}{|c|}{$R_{\mathrm{N}-\mathrm{H}} *$} & Unit & $\begin{array}{c}q_{\mathrm{HB}} \\
\left(10^{-4} \mathrm{e}\right)\end{array}$ & $\begin{array}{c}q_{\mathrm{CT}} \\
\left(10^{-4} \mathrm{e}\right)\end{array}$ & $n$ & \multicolumn{2}{|c|}{$R_{\mathrm{N}-\mathrm{H}}\left(10^{-1} \mathrm{~nm}\right) *$} & Unit & $\begin{array}{c}q_{\mathrm{HB}} \\
\left(10^{-4} \mathrm{e}\right)\end{array}$ & $\begin{array}{c}q_{\mathrm{CT}} \\
\left(10^{-4} \mathrm{e}\right)\end{array}$ \\
\hline \multirow{2}{*}{2} & $\mathbf{N}(\mathbf{1 a})-\mathbf{H}(\mathbf{1 a})$ & 1.0370 & 1 & 0 & -0.1 & \multirow{8}{*}{8} & N(1a)-H(1a) & 1.0372 & 1 & -8.7 & -9.2 \\
\hline & $\mathrm{N}(2 \mathrm{a})-\mathrm{H}(2 \mathrm{a})$ & 1.0360 & 2 & 0 & 0.2 & & $\mathbf{N}(\mathbf{2 a})-\mathbf{H}(\mathbf{2 a})$ & 1.0367 & 2 & 6.4 & 6.8 \\
\hline \multirow{3}{*}{3} & $\mathbf{N}(1 \mathbf{a})-\mathbf{H}(1 \mathbf{a})$ & 1.0371 & 1 & -7.0 & -7.0 & & $\mathbf{N}(\mathbf{3 a})-\mathbf{H}(\mathbf{3 a})$ & 1.0366 & 3 & 2.1 & 2.1 \\
\hline & $\mathbf{N}(2 \mathbf{a})-\mathbf{H}(\mathbf{2 a})$ & 1.0364 & 2 & 13.9 & 13.7 & & $\mathbf{N}(4 a)-H(4 a)$ & 1.0366 & 4 & 0.2 & 0.4 \\
\hline & $\mathrm{N}(3 \mathrm{a})-\mathrm{H}(3 \mathrm{a})$ & 1.0360 & 3 & -6.9 & -6.6 & & N(5a)-H(5a) & 1.0366 & 5 & 0 & 0.4 \\
\hline \multirow{4}{*}{4} & N(1a)-H(1a) & 1.0372 & 1 & -7.4 & -9.2 & & $\mathbf{N}(\mathbf{6 a})-\mathbf{H}(\mathbf{6 a})$ & 1.0365 & 6 & 2.3 & 2.1 \\
\hline & $\mathbf{N}(\mathbf{2 a})-\mathbf{H}(\mathbf{2 a})$ & 1.0366 & 2 & 7.4 & 9.2 & & $\mathbf{N}(7 \mathbf{a})-\mathbf{H}(7 \mathbf{a})$ & 1.0364 & 7 & 6.3 & 6.9 \\
\hline & $\mathbf{N}(3 \mathbf{a})-\mathbf{H}(\mathbf{3 a})$ & 1.0363 & 3 & 7.3 & 9.3 & & $\mathrm{~N}(8 \mathrm{a})-\mathrm{H}(8 \mathrm{a})$ & 1.0360 & 8 & -8.6 & -9.1 \\
\hline & $\mathrm{N}(4 \mathrm{a})-\mathrm{H}(4 \mathrm{a})$ & 1.0359 & 4 & -7.3 & -9.2 & \multirow{9}{*}{9} & $\mathbf{N}(1 \mathbf{a})-\mathbf{H}(1 \mathbf{a})$ & 1.0372 & 1 & -8.8 & -9.4 \\
\hline \multirow{5}{*}{5} & $\mathbf{N}(1 \mathbf{a})-\mathbf{H}(1 \mathbf{a})$ & 1.0373 & 1 & -9.0 & -9.5 & & $\mathbf{N}(2 \mathbf{a})-\mathbf{H}(2 \mathbf{a})$ & 1.0367 & 2 & 6.5 & 7.0 \\
\hline & $\mathbf{N}(\mathbf{2 a})-\mathbf{H}(\mathbf{2 a})$ & 1.0366 & 2 & 6.7 & 7.4 & & $\mathbf{N}(\mathbf{3 a})-\mathbf{H}(\mathbf{3 a})$ & 1.0366 & 3 & 2.5 & 2.4 \\
\hline & $\mathbf{N}(3 \mathbf{a})-\mathbf{H}(\mathbf{3 a})$ & 1.0366 & 3 & 4.6 & 4.5 & & $\mathbf{N}(4 a)-H(4 a)$ & 1.0366 & 4 & 0 & 0.3 \\
\hline & $\mathbf{N}(4 \mathbf{a})-\mathbf{H}(4 \mathbf{a})$ & 1.0363 & 4 & 6.7 & 7.4 & & N(5a)-H(5a) & 1.0366 & 5 & -0.4 & -0.3 \\
\hline & $\mathrm{N}(5 \mathrm{a})-\mathrm{H}(5 \mathrm{a})$ & 1.0359 & 5 & -9.0 & -9.6 & & $\mathbf{N}(6 \mathbf{6})-\mathbf{H}(\mathbf{6 a})$ & 1.0366 & 6 & 0.2 & 0.3 \\
\hline \multirow{6}{*}{6} & $\mathbf{N}(1 \mathbf{a})-\mathbf{H}(1 \mathrm{a})$ & 1.0373 & 1 & -9.0 & -9.7 & & $\mathbf{N}(7 \mathbf{a})-\mathbf{H}(\mathbf{7 a})$ & 1.0365 & 7 & 2.3 & 2.2 \\
\hline & $\mathbf{N}(2 \mathbf{a})-\mathbf{H}(2 \mathbf{a})$ & 1.0367 & 2 & 6.3 & 6.9 & & $\mathbf{N}(\mathbf{8 a})-\mathbf{H}(\mathbf{8 a})$ & 1.0363 & 8 & 6.4 & 7.0 \\
\hline & $\mathbf{N}(3 \mathbf{a})-\mathbf{H}(\mathbf{3 a})$ & 1.0366 & 3 & 2.7 & 2.9 & & $\mathrm{~N}(9 \mathrm{a})-\mathrm{H}(9 \mathrm{a})$ & 1.0359 & 9 & -8.7 & -9.2 \\
\hline & $\mathbf{N}(4 a)-H(4 a)$ & 1.0366 & 4 & 2.7 & 2.9 & \multirow{10}{*}{10} & $\mathbf{N}(\mathbf{1 a})-\mathbf{H}(\mathbf{1 a})$ & 1.0372 & 1 & -8.1 & -8.4 \\
\hline & $\mathbf{N}(5 \mathbf{a})-\mathbf{H}(5 \mathbf{a})$ & 1.0363 & 5 & 6.3 & 7.1 & & $\mathbf{N}(\mathbf{2 a})-\mathbf{H}(\mathbf{2 a})$ & 1.0367 & 2 & 6.1 & 6.7 \\
\hline & $\mathrm{N}(6 \mathrm{a})-\mathrm{H}(6 \mathrm{a})$ & 1.0359 & 6 & -9.0 & -9.9 & & $\mathbf{N}(\mathbf{3 a})-\mathbf{H}(\mathbf{3 a})$ & 1.0366 & 3 & 2.2 & 1.9 \\
\hline \multirow{7}{*}{7} & $\mathbf{N}(\mathbf{1 a})-\mathbf{H}(\mathbf{1 a})$ & 1.0372 & 1 & -9.1 & -9.9 & & $\mathbf{N}(4 a)-H(4 a)$ & 1.0366 & 4 & 0.1 & 0.2 \\
\hline & $\mathbf{N}(2 \mathbf{a})-\mathbf{H}(\mathbf{2 a})$ & 1.0367 & 2 & 6.2 & 6.8 & & N(5a)-H(5a) & 1.0366 & 5 & -0.3 & -0.3 \\
\hline & $\mathbf{N}(3 \mathbf{a})-\mathbf{H}(\mathbf{3 a})$ & 1.0366 & 3 & 2.5 & 2.6 & & $\mathbf{N}(\mathbf{6 a})-\mathbf{H}(\mathbf{6 a})$ & 1.0366 & 6 & -0.3 & -0.3 \\
\hline & $\mathbf{N}(4 a)-H(4 a)$ & 1.0366 & 4 & 6.0 & 1.2 & & $\mathbf{N}(7 \mathbf{a})-\mathbf{H}(7 \mathbf{a})$ & 1.0367 & 7 & 0.1 & 0.2 \\
\hline & $\mathbf{N}(5 \mathbf{a})-\mathbf{H}(5 \mathbf{a})$ & 1.0365 & 5 & 2.7 & 2.6 & & $\mathbf{N}(\mathbf{8 a})-\mathbf{H}(\mathbf{8 a})$ & 1.0365 & 8 & 2.2 & 1.9, \\
\hline & $\mathbf{N}(6 \mathbf{a})-\mathbf{H}(\mathbf{6 a})$ & 1.0364 & 6 & 6.2 & 7.0 & & $\mathbf{N}(9 \mathbf{a})-\mathbf{H}(9 \mathbf{a})$ & 1.0364 & 9 & 6.1 & 6.7 \\
\hline & $\mathrm{N}(7 \mathrm{a})-\mathrm{H}(7 \mathrm{a})$ & 1.0360 & 7 & -9.1 & -10.1 & & $\mathrm{~N}(10 \mathrm{a})-\mathrm{H}(10 \mathrm{a})$ & 1.0360 & 10 & -8.1 & -8.3 \\
\hline
\end{tabular}

* Since the clusters are $\mathrm{c}_{2 \mathrm{~h}}$ symmetries, only $R_{\mathrm{N}-\mathrm{H}}$ of chain "a" are listed, the $\mathrm{N}-\mathrm{H}$ bonds acting as $\mathrm{H}$-donors are displayed with bold. 
TABLE 2. $\mathrm{N} \cdots \mathrm{H}$ lengths $R_{\mathrm{N} \ldots \mathrm{H}}(\AA)$, actual charge transfer quantities along the HBs $q_{\mathrm{n} \rightarrow \sigma^{*}}\left(10^{-3}\right.$ a.u. $)$, stabilization energies $E_{n(\mathrm{~N}) \rightarrow \sigma^{*}}^{(2)}(\mathrm{kJ} / \mathrm{mol})$, electron densities $\rho\left(\mathbf{r}_{\mathrm{cp}}\right)\left(10^{-2}\right.$ a.u. $)$ and their Laplacian $\nabla^{2} \rho\left(\mathbf{r}_{\mathrm{cp}}\right)\left(10^{-2}\right.$ a.u. $)$, and local electronic potential energy density $(\mathrm{kJ} / \mathrm{mol}$ per atomic unit volume) of the ninety $\mathrm{N} \cdots \mathrm{H}$ bonds for the trans-diazene clusters $(n=2-10)$

\begin{tabular}{|c|c|c|c|c|c|c|c|c|}
\hline$n$ & $\mathrm{HB} *$ & $R_{\mathrm{N} \cdots \mathrm{H}}$ & $q_{\mathrm{n} \rightarrow \sigma^{*}}$ & $E_{n(\mathrm{~N}) \rightarrow \sigma^{*}}^{(2)}$ & $\rho\left(\mathbf{r}_{\mathrm{cp}}\right)$ & $\nabla^{2} \rho\left(\mathbf{r}_{\mathrm{cp}}\right)$ & $V_{\mathrm{A}}\left(\mathbf{r}_{\mathrm{cp}}\right)$ & $V\left(\mathbf{r}_{\mathrm{cp}}\right)$ \\
\hline \multirow{2}{*}{2} & $1 \mathrm{a} \cdots 2 \mathrm{a}$ & 2.2961 & 7.54 & 17.824 & 1.579 & .3 .991 & -23.715 & -25.988 \\
\hline & $1 b \cdots 2 b$ & 2.2958 & 7.54 & 17.824 & 1.580 & 3.993 & -23.739 & -26.005 \\
\hline \multirow{4}{*}{3} & $1 \mathrm{a} \cdots 2 \mathrm{a}$ & 2.2844 & 8.20 & 19.163 & 1.625 & 4.081 & -24.641 & -26.728 \\
\hline & $2 b \cdots 3 b$ & 2.2845 & 8.20 & 19.163 & 1.624 & 4.080 & -24.629 & -26.719 \\
\hline & $2 a \cdots 3 a$ & 2.2878 & 7.51 & 17.949 & 1.603 & 4.058 & -24.251 & -26.424 \\
\hline & $1 b \cdots 2 b$ & 2.2881 & 7.50 & 17.908 & 1.601 & 4.052 & -24.207 & -26.393 \\
\hline \multirow{6}{*}{4} & $1 \mathrm{a} \cdots 2 \mathrm{a}$ & 2.2814 & 8.16 & 19.079 & 1.636 & 4.103 & -24.873 & -26.900 \\
\hline & $3 b \cdots 4 b$ & 2.2827 & 8.13 & 18.995 & 1.632 & 4.091 & -24.780 & -26.830 \\
\hline & $2 a \cdots 3 a$ & 2.2755 & 8.02 & 18.954 & 1.652 & 4.153 & -25.244 & -27.202 \\
\hline & $2 b \cdots 3 b$ & 2.2754 & 8.02 & 18.954 & 1.652 & 4.153 & -25.247 & -27.203 \\
\hline & $3 a \cdots 4 a$ & 2.2913 & 7.39 & 17.656 & 1.594 & 4.022 & -24.013 & -26.245 \\
\hline & $1 b \cdots 2 b$ & 2.2900 & 7.43 & 17.740 & 1.598 & 4.033 & -24.103 & -26.314 \\
\hline \multirow{8}{*}{5} & $1 \mathrm{a} \cdots 2 \mathrm{a}$ & 2.2820 & 8.31 & 19.414 & 1.635 & 4.094 & -24.832 & -26.867 \\
\hline & $4 b \cdots 5 b$ & 2.2821 & 8.31 & 19.414 & 1.635 & 4.093 & -24.827 & -26.863 \\
\hline & $2 a \cdots 3 a$ & 2.2732 & 8.34 & 19.497 & 1.662 & 4.172 & -25.441 & -27.350 \\
\hline & $3 b \cdots 4 b$ & 2.2732 & 8.34 & 19.497 & 1.661 & 4.172 & -25.439 & -27.349 \\
\hline & $3 a \cdots 4 a$ & 2.2776 & 8.11 & 19.163 & 1.646 & 4.134 & -25.105 & -27.104 \\
\hline & $2 b \cdots 3 b$ & 2.2776 & 8.11 & 19.163 & 1.646 & 4.134 & -25.105 & -27.105 \\
\hline & $4 a \cdots 5 a$ & 2.2911 & 7.41 & 17.698 & 1.594 & 4.023 & -24.024 & -26.253 \\
\hline & $1 b \cdots 2 b$ & 2.2911 & 7.41 & 17.698 & 1.594 & 4.023 & -24.024 & -26.253 \\
\hline \multirow{10}{*}{6} & $1 \mathrm{a} \cdots 2 \mathrm{a}$ & 2.2820 & 8.31 & 19.414 & 1.638 & 4.066 & -24.830 & -26.802 \\
\hline & $5 b \cdots 6 b$ & 2.2822 & 8.31 & 19.414 & 1.638 & 4.064 & -24.815 & -26.792 \\
\hline & $2 a \cdots 3 a$ & 2.2728 & 8.36 & 19.539 & 1.664 & 4.193 & -25.526 & -27.450 \\
\hline & $4 b \cdots 5 b$ & 2.2727 & 8.36 & 19.539 & 1.664 & 4.193 & -25.527 & -27.449 \\
\hline & $3 a \cdots 4 a$ & 2.2753 & 8.29 & 19.372 & 1.652 & 4.157 & -25.252 & -27.214 \\
\hline & $3 b \cdots 4 b$ & 2.2752 & 8.29 & 19.372 & 1.652 & 4.158 & -25.261 & -27.221 \\
\hline & $4 a \cdots 5 a$ & 2.2782 & 8.09 & 19.121 & 1.647 & 4.116 & -25.076 & -27.057 \\
\hline & $2 b \cdots 3 b$ & 2.2781 & 8.09 & 19.121 & 1.647 & 4.117 & -25.089 & -27.066 \\
\hline & $5 a \cdots 6 a$ & 2.2915 & 7.41 & 17.698 & 1.589 & 4.032 & -23.961 & -26.224 \\
\hline & $1 b \cdots 2 b$ & 2.2917 & 7.41 & 17.698 & 1.588 & 4.031 & -23.954 & -26.220 \\
\hline \multirow{12}{*}{7} & $1 \mathrm{a} \cdots 2 \mathrm{a}$ & 2.2807 & 8.34 & 19.497 & 1.641 & 4.106 & -24.967 & -27.010 \\
\hline & $6 b \cdots 7 b$ & 2.2810 & 8.34 & 19.497 & 1.645 & 4.085 & -24.972 & -26.961 \\
\hline & $2 \mathrm{a} \cdots 3 \mathrm{a}$ & 2.2728 & 8.36 & 19.539 & 1.674 & 4.115 & -25.524 & -27.303 \\
\hline & $5 b \cdots 6 b$ & 2.2727 & 8.36 & 19.539 & 1.674 & 4.132 & -25.554 & -27.341 \\
\hline & $3 a \cdots 4 a$ & 2.2751 & 8.29 & 19.372 & 1.668 & 4.081 & -25.347 & -27.098 \\
\hline & $4 b \cdots 5 b$ & 2.2750 & 8.31 & 19.414 & 1.662 & 4.120 & -25.336 & -27.150 \\
\hline & $4 a \cdots 5 a$ & 2.2758 & 8.27 & 19.330 & 1.646 & 4.163 & -25.159 & -27.110 \\
\hline & $3 b \cdots 4 b$ & 2.2757 & 8.27 & 19.330 & 1.638 & 4.228 & -25.172 & -27.250 \\
\hline & $5 a \cdots 6 a$ & 2.2786 & 8.07 & 19.079 & 1.638 & 4.167 & -25.041 & -27.130 \\
\hline & $2 b \cdots 3 b$ & 2.2785 & 8.07 & 19.079 & 1.630 & 4.179 & -24.938 & -27.092 \\
\hline & $6 a \cdots 7 a$ & 2.2906 & 7.43 & 17.740 & 1.592 & 4.054 & -24.067 & -26.340 \\
\hline & $1 b \cdots 2 b$ & 2.2908 & 7.43 & 17.740 & 1.592 & 4.039 & -24.029 & -26.310 \\
\hline \multirow[t]{7}{*}{8} & $1 \mathrm{a} \cdots 2 \mathrm{a}$ & 2.2838 & 8.25 & 19.288 & 1.628 & 4.083 & -24.704 & -26.776 \\
\hline & $7 b \cdots 8 b$ & 2.2819 & 8.31 & 19.414 & 1.635 & 4.103 & -24.860 & -26.899 \\
\hline & $2 a \cdots 3 a$ & 2.2734 & 8.34 & 19.497 & 1.658 & 4.167 & -25.374 & -27.291 \\
\hline & $6 b \cdots 7 b$ & 2.2734 & 8.34 & 19.497 & 1.658 & 4.167 & -25.375 & -27.292 \\
\hline & $3 a \cdots 4 a$ & 2.2753 & 8.29 & 19.372 & 1.652 & 4.150 & -25.241 & -27.196 \\
\hline & $5 b \cdots 6 b$ & 2.2752 & 8.29 & 19.372 & 1.653 & 4.152 & -25.254 & -27.205 \\
\hline & $4 a \cdots 5 a$ & 2.2754 & 8.29 & 19.372 & 1.652 & 4.149 & -25.235 & -27.189 \\
\hline
\end{tabular}




\begin{tabular}{|c|c|c|c|c|c|c|c|c|}
\hline & $4 b \cdots 5 b$ & 2.2753 & 8.29 & 19.372 & 1.653 & 4.151 & -25.249 & -27.199 \\
\hline & $5 a \cdots 6 a$ & 2.2755 & 8.27 & 19.330 & 1.652 & 4.149 & -25.233 & -27.186 \\
\hline & $3 b \cdots 4 b$ & 2.2752 & 8.29 & 19.372 & 1.653 & 4.152 & -25.253 & -27.200 \\
\hline & $6 a \cdots 7 a$ & 2.2777 & 8.11 & 19.163 & 1.644 & 4.131 & -25.058 & -27.055 \\
\hline & $2 b \cdots 3 b$ & 2.2773 & 8.11 & 19.163 & 1.645 & 4.135 & -25.089 & -27.077 \\
\hline & $7 a \cdots 8 a$ & 2.2901 & 7.44 & 17.782 & 1.594 & 4.039 & -24.056 & -26.283 \\
\hline & $1 b \cdots 2 b$ & 2.2919 & 7.39 & 17.656 & 1.587 & 4.020 & -23.912 & -26.168 \\
\hline \multirow{16}{*}{9} & $1 \mathrm{a} \cdots 2 \mathrm{a}$ & 2.2815 & 8.33 & 19.456 & 1.637 & 4.107 & -24.891 & -26.924 \\
\hline & $8 b \cdots 9 b$ & 2.2820 & 8.31 & 19.414 & 1.635 & 4.101 & -24.846 & -26.887 \\
\hline & $2 a \cdots 3 a$ & 2.2736 & 8.34 & 19.497 & 1.658 & 4.165 & -25.360 & -27.281 \\
\hline & $7 b \cdots 8 b$ & 2.2734 & 8.34 & 19.497 & 1.658 & 4.167 & -25.372 & -27.291 \\
\hline & $3 a \cdots 4 a$ & 2.2756 & 8.29 & 19.372 & 1.651 & 4.148 & -25.221 & -27.182 \\
\hline & $6 b \cdots 7 b$ & 2.2758 & 8.27 & 19.330 & 1.651 & 4.146 & -25.208 & -27.172 \\
\hline & $4 a \cdots 5 a$ & 2.2757 & 8.27 & 19.330 & 1.651 & 4.147 & -25.216 & -27.176 \\
\hline & $5 b \cdots 6 b$ & 2.2759 & 8.27 & 19.330 & 1.651 & 4.145 & -25.204 & -27.167 \\
\hline & $5 a \cdots 6 a$ & 2.2751 & 8.29 & 19.372 & 1.653 & 4.152 & -25.258 & -27.205 \\
\hline & $4 b \cdots 5 b$ & 2.2753 & 8.29 & 19.372 & 1.653 & 4.150 & -25.245 & -27.195 \\
\hline & $6 a \cdots 7 a$ & 2.2750 & 8.29 & 19.372 & 1.654 & 4.153 & -25.267 & -27.210 \\
\hline & $3 b \cdots 4 b$ & 2.2752 & 8.29 & 19.372 & 1.653 & 4.151 & -25.253 & -27.199 \\
\hline & $7 a \cdots 8 a$ & 2.2776 & 8.11 & 19.163 & 1.644 & 4.132 & -25.069 & -27.063 \\
\hline & $2 b \cdots 3 b$ & 2.2776 & 8.11 & 19.163 & 1.644 & 4.132 & -25.068 & -27.061 \\
\hline & $8 a \cdots 9 a$ & 2.2906 & 7.43 & 17.740 & 1.592 & 4.033 & -24.007 & -26.244 \\
\hline & $1 b \cdots 2 b$ & 2.2898 & 7.46 & 17.824 & 1.595 & 4.042 & -24.074 & -26.298 \\
\hline \multirow{18}{*}{10} & $1 \mathrm{a} \cdots 2 \mathrm{a}$ & 2.2816 & 8.29 & 19.372 & 1.631 & 4.083 & -24.754 & -26.781 \\
\hline & $9 b \cdots 10 b$ & 2.2816 & 8.29 & 19.372 & 1.631 & 4.084 & -24.756 & -26.782 \\
\hline & $2 a \cdots 3 a$ & 2.2745 & 8.31 & 19.414 & 1.656 & 4.160 & -25.326 & -27.261 \\
\hline & $8 b \cdots 9 b$ & 2.2745 & 8.31 & 19.414 & 1.656 & 4.160 & -25.328 & -27.263 \\
\hline & $3 a \cdots 4 a$ & 2.2763 & 8.25 & 19.288 & 1.650 & 4.143 & -25.183 & -27.156 \\
\hline & $7 b \cdots 8 b$ & 2.2763 & 8.25 & 19.288 & 1.650 & 4.143 & -25.185 & -27.157 \\
\hline & $4 a \cdots 5 a$ & 2.2766 & 8.24 & 19.246 & 1.649 & 4.140 & -25.160 & -27.136 \\
\hline & $6 b \cdots 7 b$ & 2.2765 & 8.24 & 19.246 & 1.649 & 4.140 & -25.162 & -27.137 \\
\hline & $5 a \cdots 6 a$ & 2.2762 & 8.25 & 19.288 & 1.650 & 4.143 & -25.184 & -27.151 \\
\hline & $5 b \cdots 6 b$ & 2.2762 & 8.25 & 19.288 & 1.650 & 4.143 & -25.186 & -27.153 \\
\hline & $6 a \cdots 7 a$ & 2.2756 & 8.27 & 19.330 & 1.651 & 4.148 & -25.220 & -27.176 \\
\hline & $4 b \cdots 5 b$ & 2.2756 & 8.27 & 19.330 & 1.651 & 4.148 & -25.222 & -27.177 \\
\hline & $7 \mathrm{a} \cdots 8 \mathrm{a}$ & 2.2755 & 8.27 & 19.330 & 1.651 & 4.150 & -25.222 & -27.178 \\
\hline & $3 b \cdots 4 b$ & 2.2755 & 8.27 & 19.330 & 1.651 & 4.150 & -25.223 & -27.178 \\
\hline & $8 a \cdots 9 a$ & 2.2773 & 8.11 & 19.163 & 1.644 & 4.135 & -25.075 & -27.068 \\
\hline & $2 b \cdots 3 b$ & 2.2773 & 8.11 & 19.163 & 1.644 & 4.135 & -25.076 & -27.069 \\
\hline & $9 a \cdots 10 a$ & 2.2865 & 7.48 & 17.866 & 1.611 & 4.048 & -24.347 & -26.459 \\
\hline & $1 b \cdots 2 b$ & 2.2865 & 7.48 & 17.866 & 1.611 & 4.048 & -24.349 & -26.460 \\
\hline
\end{tabular}

* In this column, the labels of the $\mathrm{N}$ and $\mathrm{H}$ atoms of the $\mathrm{N} \cdots \mathrm{H}$ bonds that are given in the figure 1 of the text are listed.

Cartesian coordinates (in angstroms) for B3LYP/6-31++G** optimized geometries

Isolated molecule $(n=1)$ :

$\begin{array}{llll}\mathrm{N} & 0.2710989022 & 0.3427531856 & -0.4427578293 \\ \mathrm{~N} & -0.2709323568 & -0.3428709313 & 0.4427686502 \\ \mathrm{H} & 0.0224205748 & -0.090978764 & -1.3504872173 \\ \mathrm{H} & -0.0235863926 & 0.0918029839 & 1.3504114711\end{array}$

$\operatorname{Dimer}(n=2)$ :

$\begin{array}{llll}\mathrm{N} & 0.9903803036 & 1.3361779598 & 0.7154347691 \\ \mathrm{~N} & 0.3507584201 & 0.5591288648 & 1.4483480865 \\ \mathrm{H} & 0.8052759288 & 1.0266781713 & -0.2568081424\end{array}$




$\begin{array}{llll}\mathrm{H} & 0.5173275974 & 0.8454698876 & 2.4299190408 \\ \mathrm{~N} & -0.3502054102 & -0.5594850362 & -1.4482350065 \\ \mathrm{~N} & -0.9909029422 & -1.3358415592 & -0.7155469389 \\ \mathrm{H} & -0.5161765925 & -0.8459644415 & -2.4298715032 \\ \mathrm{H} & -0.8066395331 & -1.0260452226 & 0.2567542337\end{array}$

\begin{tabular}{cccc}
\multicolumn{4}{c}{ Trimer $(n=3)$ : } \\
$\mathrm{N}$ & 0.2842469693 & 0.4392773442 & -0.3397957559 \\
$\mathrm{~N}$ & -0.2866279304 & -0.4365913068 & 0.3384217996 \\
$\mathrm{H}$ & 0.2051099616 & 0.1481857865 & -1.3313631076 \\
$\mathrm{H}$ & -0.2073639808 & -0.1454426465 & 1.3299545498 \\
$\mathrm{~N}$ & -0.6503597892 & -1.5284260231 & -2.6323848539 \\
$\mathrm{~N}$ & -1.2206057032 & -2.4065364357 & -1.9585818592 \\
$\mathrm{H}$ & -0.7186899527 & -1.8041083533 & -3.6286419537 \\
$\mathrm{H}$ & -1.1368036325 & -2.1053368381 & -0.9696983998 \\
$\mathrm{~N}$ & 1.2228563824 & 2.4047163084 & 1.959572768 \\
$\mathrm{~N}$ & 0.6504739829 & 1.5275400341 & 2.6327785586 \\
$\mathrm{H}$ & 1.1368934523 & 2.1053247043 & 0.970320465 \\
$\mathrm{H}$ & 0.7209667693 & 1.8015178996 & 3.6293538456
\end{tabular}

Tetramer $(n=4)$ :

$\begin{array}{lllc}\mathrm{N} & 1.4733713544 & -0.610309126 & 0.0006829925 \\ \mathrm{~N} & 1.6824417037 & 0.6183321415 & 0.0008518716 \\ \mathrm{H} & 2.4019401764 & -1.0703866374 & 0.0007244497 \\ \mathrm{H} & 0.7543057027 & 1.0799807199 & 0.0008486578 \\ \mathrm{~N} & 4.6305557195 & -0.5984771115 & 0.0007510982 \\ \mathrm{~N} & 4.8471211722 & 0.6273587384 & 0.0005765395 \\ \mathrm{H} & 5.5482760204 & -1.0786508521 & 0.0004759187 \\ \mathrm{H} & 3.9143168071 & 1.0815853209 & 0.0007092535 \\ \mathrm{~N} & -1.6821237813 & -0.617300421 & -0.0003766902 \\ \mathrm{~N} & -1.4735657515 & 0.6114313656 & 0.0000562265 \\ \mathrm{H} & -0.7537565371 & -1.0784958118 & -0.0001430408 \\ \mathrm{H} & -2.4023032454 & 1.0711555639 & -0.0001009769 \\ \mathrm{~N} & -4.8466732951 & -0.6285348064 & -0.0015231154 \\ \mathrm{~N} & -4.6311344631 & 0.5974823122 & -0.0010715476 \\ \mathrm{H} & -3.9134790403 & -1.0819604433 & -0.0012421699 \\ \mathrm{H} & -5.5492484967 & 1.0768904915 & -0.0009037181\end{array}$

\begin{tabular}{cclc}
\multicolumn{4}{c}{ Pentamer $(n=5):$} \\
$\mathrm{N}$ & 3.0493143593 & -0.6171381714 & 0.0005117477 \\
$\mathrm{~N}$ & 3.2609070128 & 0.6110552166 & 0.000888913 \\
$\mathrm{H}$ & 3.9764761925 & -1.08002517 & 0.0006625024 \\
$\mathrm{H}$ & 2.3333561467 & 1.0739467032 & 0.0007603216 \\
$\mathrm{~N}$ & 6.2147613971 & -0.5907475042 & 0.0014168862 \\
$\mathrm{~N}$ & 6.4271804896 & 0.6360047358 & 0.0018212334 \\
$\mathrm{H}$ & 7.1341622444 & -1.0680390395 & 0.0015849098 \\
$\mathrm{H}$ & 5.4929115367 & 1.0866174923 & 0.0016225002 \\
$\mathrm{~N}$ & -0.1072391356 & -0.6138676502 & -0.0002631555 \\
$\mathrm{~N}$ & 0.1072128483 & 0.613918475 & 0.0000918271 \\
$\mathrm{H}$ & 0.8190405678 & -1.0791105221 & -0.0001551125 \\
$\mathrm{H}$ & -0.8190670375 & 1.0791601858 & 0.0000163171 \\
$\mathrm{~N}$ & -6.4271222847 & -0.6360692717 & -0.0016401089 \\
$\mathrm{~N}$ & -6.2147666993 & 0.5906930714 & -0.0012585797 \\
$\mathrm{H}$ & -5.4928277393 & -1.0866309201 & -0.0015252197
\end{tabular}




$\begin{array}{llll}\mathrm{H} & -7.1341880891 & 1.0679405446 & -0.0013298278 \\ \mathrm{~N} & -3.2609161159 & -0.6110227179 & -0.0009642393 \\ \mathrm{~N} & -3.0493329233 & 0.617172346 & -0.000616796 \\ \mathrm{H} & -2.3333602958 & -1.0739057623 & -0.000876619 \\ \mathrm{H} & -3.9764961639 & 1.080056783 & -0.0006738683\end{array}$

Hexamer $(n=6)$ :

$\begin{array}{llll}\mathrm{N} & 7.7934816766 & -0.5857515213 & 0.0011642104 \\ \mathrm{~N} & 8.0040173151 & 0.6413193327 & 0.0014738037 \\ \mathrm{H} & 8.7136239923 & -1.0616244106 & 0.0009027689 \\ \mathrm{H} & 7.0690606299 & 1.0905274773 & 0.0013222295 \\ \mathrm{~N} & 1.4707174892 & -0.6163868287 & -0.0002419458 \\ \mathrm{~N} & 1.6842286041 & 0.6115741438 & 0.000022642 \\ \mathrm{H} & 2.3973292115 & -1.0809502015 & -0.0001739841 \\ \mathrm{H} & 0.7574921881 & 1.0759664941 & -0.0000330941 \\ \mathrm{~N} & 4.6277663981 & -0.616857187 & 0.0003204411 \\ \mathrm{~N} & 4.8376975422 & 0.6116205206 & 0.0006524925 \\ \mathrm{H} & 5.5555668186 & -1.0784408129 & 0.0004282642 \\ \mathrm{H} & 3.909529155 & 1.0733039981 & 0.0005265632 \\ \mathrm{~N} & -1.6842945123 & -0.6118961156 & -0.0004999895 \\ \mathrm{~N} & -1.4707133031 & 0.6160534163 & -0.0003192144 \\ \mathrm{H} & -0.7575878714 & -1.0763490467 & -0.0005043458 \\ \mathrm{H} & -2.3973020475 & 1.0806616261 & -0.0003027403 \\ \mathrm{~N} & -8.0039703725 & -0.6410643309 & -0.000766303 \\ \mathrm{~N} & -7.7934636108 & 0.5860066047 & -0.0005968653 \\ \mathrm{H} & -7.068996324 & -1.0902422272 & -0.0007590465 \\ \mathrm{H} & -8.7135974492 & 1.06187545 & -0.0007789531 \\ \mathrm{~N} & -4.8378048376 & -0.6115339768 & -0.0006386048 \\ \mathrm{~N} & -4.6276682373 & 0.6169078096 & -0.0004977401 \\ \mathrm{H} & -3.9097049041 & -1.0733553368 & -0.0006622569 \\ \mathrm{H} & -5.5553724608 & 1.0786839189 & -0.0004758926\end{array}$

$\begin{array}{cccc}\text { Heptamer }(n=7): & & \\ \mathrm{N} & 9.370703758 & -0.5801045547 & 0.0012001031 \\ \mathrm{~N} & 9.579918959 & 0.6473317947 & 0.0015889781 \\ \mathrm{H} & 10.291433893 & -1.0549933423 & 0.0009962649 \\ \mathrm{H} & 8.6444503827 & 1.0951946786 & 0.001484813 \\ \mathrm{~N} & 3.0488853998 & -0.6176922299 & 0.0000786427 \\ \mathrm{~N} & 3.2615686007 & 0.610402648 & 0.0003545912 \\ \mathrm{H} & 3.9758148626 & -1.0815912892 & 0.0000904378 \\ \mathrm{H} & 2.3345233716 & 1.0742021413 & 0.0003225427 \\ \mathrm{~N} & 6.2063344818 & -0.6161398333 & 0.000517704 \\ \mathrm{~N} & 6.4151264416 & 0.612561195 & 0.0008818511 \\ \mathrm{H} & 7.1346453842 & -1.0769039481 & 0.0005584991 \\ \mathrm{H} & 5.486597126 & 1.0735298523 & 0.0008152104 \\ \mathrm{~N} & -0.1066228241 & -0.61428014 & -0.0001631648 \\ \mathrm{~N} & 0.1066229321 & 0.6137402962 & 0.0000237069 \\ \mathrm{H} & 0.820189839 & -1.0785013352 & -0.0001651944 \\ \mathrm{H} & -0.8201929827 & 1.0779557306 & -0.0000007918 \\ \mathrm{~N} & -6.415245982 & -0.6123425383 & -0.0007672714 \\ \mathrm{~N} & -6.2062208899 & 0.6163162369 & -0.0005865923 \\ \mathrm{H} & -5.4867977581 & -1.0734716116 & -0.0007201757 \\ \mathrm{H} & -7.1344239228 & 1.0772984265 & -0.0006794025 \\ \mathrm{~N} & -3.2616387244 & -0.6107311323 & -0.0004229258\end{array}$




$\begin{array}{llll}\mathrm{N} & -3.0488141303 & 0.6173396561 & -0.0002669307 \\ \mathrm{H} & -2.3346498594 & -1.07464195 & -0.0004083672 \\ \mathrm{H} & -3.9756907002 & 1.0813451047 & -0.0003124074 \\ \mathrm{~N} & -9.5798772155 & -0.646930725 & -0.0012774625 \\ \mathrm{~N} & -9.3707438848 & 0.580521147 & -0.0010621356 \\ \mathrm{H} & -8.6443682931 & -1.0947092828 & -0.0012081621 \\ \mathrm{H} & -10.2915097988 & 1.0553440829 & -0.0014669251\end{array}$

\begin{tabular}{|c|c|c|c|}
\hline \multicolumn{4}{|c|}{ Octamer $(n=8)$ : } \\
\hline $\mathrm{N}$ & 10.9486646644 & -0.5707587508 & 0.0009726618 \\
\hline $\mathrm{N}$ & 11.1580073055 & 0.6566328578 & 0.0012445259 \\
\hline $\mathrm{H}$ & 11.8693565444 & -1.0456876776 & 0.000923807 \\
\hline $\mathrm{H}$ & 10.2226230878 & 1.1046977776 & 0.001142336 \\
\hline $\mathrm{N}$ & 7.7846022662 & -0.6072474585 & 0.0004043759 \\
\hline $\mathrm{N}$ & 7.992487796 & 0.6216018231 & 0.0006748793 \\
\hline $\mathrm{H}$ & 8.7131301651 & -1.0675666025 & 0.0004531112 \\
\hline $\mathrm{H}$ & 7.0635030631 & 1.0816328423 & 0.0005975551 \\
\hline $\mathrm{N}$ & 1.4724167574 & -0.6146638134 & -0.0003938108 \\
\hline $\mathrm{N}$ & 1.6832886705 & 0.6137691015 & -0.0002183178 \\
\hline $\mathrm{H}$ & 2.4001396949 & -1.077066654 & -0.0003301701 \\
\hline $\mathrm{H}$ & 0.755561806 & 1.076196185 & -0.0002559226 \\
\hline $\mathrm{N}$ & 4.6276815283 & -0.6124812857 & -0.0000676861 \\
\hline $\mathrm{N}$ & 4.8383313456 & 0.6159762496 & 0.0001601453 \\
\hline $\mathrm{H}$ & 5.5554082612 & -1.0747976368 & 0.0000065691 \\
\hline $\mathrm{H}$ & 3.9105264607 & 1.0782718722 & 0.000088188 \\
\hline $\mathrm{N}$ & -1.6828523916 & -0.6163260099 & -0.0005111676 \\
\hline $\mathrm{N}$ & -1.4718519877 & 0.6120836792 & -0.0003899984 \\
\hline $\mathrm{H}$ & -0.7551750994 & -1.0788521714 & -0.0005363167 \\
\hline $\mathrm{H}$ & -2.39953117 & 1.0745712797 & -0.0003268715 \\
\hline $\mathrm{N}$ & -7.9921516984 & -0.6223068561 & -0.000412227 \\
\hline $\mathrm{N}$ & -7.7834394366 & 0.6063792073 & -0.0002218778 \\
\hline $\mathrm{H}$ & -7.0633270577 & -1.0826543697 & -0.0004586173 \\
\hline $\mathrm{H}$ & -8.7114613344 & 1.0674938548 & -0.0001381326 \\
\hline $\mathrm{N}$ & -4.8379948291 & -0.6177252197 & -0.0004814273 \\
\hline $\mathrm{N}$ & -4.6269277964 & 0.6106719943 & -0.0003509753 \\
\hline $\mathrm{H}$ & -3.9103648529 & -1.0803769272 & -0.0005490198 \\
\hline $\mathrm{H}$ & -5.5545144961 & 1.0732911241 & -0.0002378408 \\
\hline $\mathrm{N}$ & -11.1602792706 & -0.6513597837 & -0.0003342617 \\
\hline $\mathrm{N}$ & -10.9500107956 & 0.5757698301 & -0.0000727377 \\
\hline $\mathrm{H}$ & -10.2253384518 & -1.1005097118 & -0.0003767995 \\
\hline $\mathrm{H}$ & -11.8703415181 & 1.05124786 & -0.000016581 \\
\hline
\end{tabular}

\begin{tabular}{clcc}
\multicolumn{4}{c}{ Enneamer $(n=9):$} \\
$\mathrm{N}$ & 12.5268739933 & -0.5715433287 & 0.000535739 \\
$\mathrm{~N}$ & 12.736420214 & 0.655737149 & 0.0005936152 \\
$\mathrm{H}$ & 13.4474212476 & -1.0466879981 & 0.0007026222 \\
$\mathrm{H}$ & 11.8011250215 & 1.1041551481 & 0.0005506804 \\
$\mathrm{~N}$ & 6.205010849 & -0.608804317 & -0.0001087486 \\
$\mathrm{~N}$ & 6.416191295 & 0.6195588304 & -0.0000431999 \\
$\mathrm{H}$ & 7.1325082598 & -1.0715825956 & -0.0000769426 \\
$\mathrm{H}$ & 5.4885874828 & 1.0822544523 & -0.0000731598 \\
$\mathrm{~N}$ & 9.3617860082 & -0.6051472086 & 0.0001116758 \\
$\mathrm{~N}$ & 9.570271534 & 0.6235910365 & 0.0002189629
\end{tabular}




\begin{tabular}{|c|c|c|c|}
\hline $\mathrm{H}$ & 10.2901680345 & -1.0656186867 & 0.0002071701 \\
\hline $\mathrm{H}$ & 8.6416235622 & 1.0843203693 & 0.000132147 \\
\hline $\mathrm{N}$ & 3.0499758751 & -0.610390625 & -0.0000840828 \\
\hline $\mathrm{N}$ & 3.260647176 & 0.618078319 & -0.0000623217 \\
\hline $\mathrm{H}$ & 3.9777809091 & -1.072620599 & -0.0001213458 \\
\hline $\mathrm{H}$ & 2.332854018 & 1.0803754393 & -0.0000240085 \\
\hline $\mathrm{N}$ & -3.2607060803 & -0.6164208334 & 0.000067976 \\
\hline $\mathrm{N}$ & -3.0502796103 & 0.6120889275 & 0.0001881385 \\
\hline $\mathrm{H}$ & -2.3328160547 & -1.0785248827 & 0.0000405344 \\
\hline $\mathrm{H}$ & -3.9781702105 & 1.074147857 & 0.0002136372 \\
\hline $\mathrm{N}$ & -0.105178678 & -0.613274131 & 0.000024174 \\
\hline $\mathrm{N}$ & 0.1050036786 & 0.6152809357 & 0.0000622329 \\
\hline $\mathrm{H}$ & 0.8228153452 & -1.0751553239 & -0.000035299 \\
\hline $\mathrm{H}$ & -0.8229878109 & 1.0771659733 & 0.0001222459 \\
\hline $\mathrm{N}$ & -6.4161022055 & -0.6189249383 & -0.0000667071 \\
\hline $\mathrm{N}$ & -6.2054403586 & 0.6095285109 & 0.0001354291 \\
\hline $\mathrm{H}$ & -5.4883248355 & -1.0812767803 & -0.0000644229 \\
\hline $\mathrm{H}$ & -7.1331711643 & 1.0718273981 & 0.0001329486 \\
\hline $\mathrm{N}$ & -12.7355781108 & -0.6587841474 & -0.0007456956 \\
\hline $\mathrm{N}$ & -12.5261533367 & 0.5685953587 & -0.0003841603 \\
\hline $\mathrm{H}$ & -11.8002179585 & -1.106918329 & -0.0006281128 \\
\hline $\mathrm{H}$ & -13.446814097 & 1.0435839699 & -0.0003865303 \\
\hline $\mathrm{N}$ & -9.5704058543 & -0.6240155504 & -0.0003466763 \\
\hline $\mathrm{N}$ & -9.3623355657 & 0.6048016221 & -0.0001294523 \\
\hline $\mathrm{H}$ & -8.6415453463 & -1.0843031076 & -0.0003730669 \\
\hline $\mathrm{H}$ & -10.2908421641 & 1.0651684255 & -0.000087389 \\
\hline \multicolumn{4}{|c|}{ Decamer $(n=10)$ : } \\
\hline $\mathrm{N}$ & 14.1026878225 & -0.5648310099 & -0.0011196858 \\
\hline $\mathrm{N}$ & 14.3143656455 & 0.6621571823 & -0.0010901412 \\
\hline $\mathrm{H}$ & 15.0224232299 & -1.0415969713 & -0.001470704 \\
\hline $\mathrm{H}$ & 13.3798037747 & 1.1119783531 & -0.000771214 \\
\hline $\mathrm{N}$ & 10.9411023363 & -0.5972324256 & 0.0000008417 \\
\hline $\mathrm{N}$ & 11.1493989775 & 0.631551885 & -0.0002904442 \\
\hline $\mathrm{H}$ & 11.8698123897 & -1.0572277968 & -0.0003111622 \\
\hline $\mathrm{H}$ & 10.220890469 & 1.092574635 & -0.0000642123 \\
\hline $\mathrm{N}$ & 4.6288511805 & -0.6058562869 & 0.0013986931 \\
\hline $\mathrm{N}$ & 4.838774717 & 0.6227278788 & 0.0015939067 \\
\hline $\mathrm{H}$ & 5.5569271574 & -1.0675241187 & 0.0013802136 \\
\hline $\mathrm{H}$ & 3.9107225921 & 1.0845022964 & 0.0016503661 \\
\hline $\mathrm{N}$ & 7.7842864622 & -0.6019554626 & 0.0013677896 \\
\hline $\mathrm{N}$ & 7.9946827819 & 0.6265326921 & 0.0010912054 \\
\hline $\mathrm{H}$ & 8.7121730728 & -1.0639135316 & 0.0011228456 \\
\hline $\mathrm{H}$ & 7.0668663152 & 1.0888054261 & 0.0013189974 \\
\hline $\mathrm{N}$ & 1.4732939297 & -0.6113607192 & 0.0003183769 \\
\hline $\mathrm{N}$ & 1.6826310115 & 0.6173258318 & 0.0008250062 \\
\hline $\mathrm{H}$ & 2.4015955198 & -1.0726030171 & 0.0005405024 \\
\hline $\mathrm{H}$ & 0.7543425595 & 1.0786105554 & 0.0006547014 \\
\hline $\mathrm{N}$ & -4.8387943717 & -0.6229650093 & -0.0017085203 \\
\hline $\mathrm{N}$ & -4.6288206791 & 0.6056105599 & -0.0014323242 \\
\hline $\mathrm{H}$ & -3.9107618631 & -1.0847785512 & -0.0016914048 \\
\hline $\mathrm{H}$ & -5.5568783364 & 1.0673152028 & -0.0013135447 \\
\hline $\mathrm{N}$ & -1.6826323296 & -0.6176527592 & -0.0008662092 \\
\hline $\mathrm{N}$ & -1.4732780891 & 0.6110308569 & -0.0003418396 \\
\hline
\end{tabular}




$\begin{array}{llll}\mathrm{H} & -0.7543510989 & -1.0789517577 & -0.000620643 \\ \mathrm{H} & -2.4015739325 & 1.0722848667 & -0.0005084045 \\ \mathrm{~N} & -7.994720602 & -0.6265984375 & -0.001256229 \\ \mathrm{~N} & -7.7842449701 & 0.6018762032 & -0.001432573 \\ \mathrm{H} & -7.0669350604 & -1.0889329031 & -0.0014912854 \\ \mathrm{H} & -8.7121026457 & 1.0638921856 & -0.0011273657 \\ \mathrm{~N} & -14.3144466559 & -0.661672992 & 0.0018207412 \\ \mathrm{~N} & -14.1026247407 & 0.5652923922 & 0.0009201789 \\ \mathrm{H} & -13.3799335639 & -1.111592125 & 0.0010971132 \\ \mathrm{H} & -15.0222982129 & 1.0421796057 & 0.0013358577 \\ \mathrm{~N} & -11.1494582696 & -0.6313748374 & 0.0002491142 \\ \mathrm{~N} & -10.9410554499 & 0.5973917109 & -0.0000180908 \\ \mathrm{H} & -10.2209893931 & -1.0924767482 & -0.0000259483 \\ \mathrm{H} & -11.8697239223 & 1.0574736197 & 0.0000867146\end{array}$

\section{Complete references}

(26) Frisch, M. J; Trucks, G. W.; Schlegel, H. B.; Scuseria, G. E.; Robb, M. A.; Cheeseman, J. R.; Montgomery, J. A., Jr.; Vreven, T.; Kudin, K. N.; Burant, J. C.; Millam, J. M.; Iyengar, S. S.; Tomasi, J.; Barone, V.; Mennucci, B.; Cossi, M.; Scalmani, G.; Rega, N.; Petersson, G. A.; Nakatsuji, H.; Hada, M.; Ehara, M.; Toyota, K. Fukuda, R.; Hasegawa, J.; Ishida, M.; Nakajima, T.; Honda, Y.; Kitao, O.; Nakai, H.; Klene, M.; Li, X.; Knox, J. E.; Hratchian, H. P.; Cross, J. B.; Adamo, C.; Jaramillo, J.; Gomperts, R.; Stratmann, R. E.; Yazyev, O.; Austin, A. J.; Cammi, R.; Pomelli, C.; Ochterski, J. W.; Ayala, P. Y.; Morokuma, K.; Voth, G. A.; Salvador, P.; Dannenberg, J. J.; Zakrzewski, V. G.; Dapprich, S.; Daniels, A. D.; Strain, M. C.; Farkas, O.; Malick, D. K.; Rabuck, A. D.; Raghavachari, K.; Foresman, J. B.; Ortiz, J. V.; Cui, Q.; Baboul, A. G.; Clifford, S.; Cioslowski, J.; Stefanov, B. B.; Liu, G.; Liashenko, A.; Piskorz, P.; Komaromi, I.; Martin, R. L.; Fox, D. J.; Keith, T.; Al-Laham, M. A.; Peng, C. Y.; Nanayakkara, A.; Challacombe, M.; Gill, P. M. W.; Johnson, B.; Chen, W.; Wong, M. W.; Gonzalez, C.; Pople, J. A. Gaussian 03, Revision B.05; Gaussian, Inc.: Pittsburgh PA, 2003. 